\title{
Cardiac macrophage migration inhibitory factor inhibits JNK pathway activation and injury during ischemia/reperfusion
}

\author{
Dake Qi, ${ }^{1}$ Xiaoyue Hu, ${ }^{1}$ Xiaohong Wu, ${ }^{1}$ Melanie Merk, ${ }^{2}$ \\ Lin Leng, ${ }^{2}$ Richard Bucala, ${ }^{2}$ and Lawrence H. Young ${ }^{1}$ \\ ${ }^{1}$ Section of Cardiovascular Medicine and ${ }^{2}$ Section of Rheumatology, Department of Internal Medicine, \\ Yale University School of Medicine, New Haven, Connecticut, USA.
}

\begin{abstract}
Macrophage migration inhibitory factor (MIF) is a proinflammatory cytokine that also modulates physiologic cell signaling pathways. MIF is expressed in cardiomyocytes and limits cardiac injury by enhancing AMPK activity during ischemia. Reperfusion injury is mediated in part by activation of the stress kinase JNK, but whether MIF modulates JNK in this setting is unknown. We examined the role of MIF in regulating JNK activation and cardiac injury during experimental ischemia/reperfusion in mouse hearts. Isolated perfused $\mathrm{Mif}^{/-}$hearts had greater contractile dysfunction, necrosis, and JNK activation than WT hearts, with increased upstream MAPK kinase 4 phosphorylation, following ischemia/reperfusion. These effects were reversed if recombinant MIF was present during reperfusion, indicating that MIF deficiency during reperfusion exacerbated injury. Activated JNK acts in a proapoptotic manner by regulating BCL2-associated agonist of cell death (BAD) phosphorylation, and this effect was accentuated in $M i f /-$ hearts after ischemia/reperfusion. Similar detrimental effects of MIF deficiency were observed in vivo following coronary occlusion and reperfusion in $\mathrm{Mif}^{/-}$mice. Importantly, excess JNK activation also was observed after hypoxia-reoxygenation in human fibroblasts homozygous for the MIF allele with the lowest level of promoter activity. These data indicate that endogenous MIF inhibits JNK pathway activation during reperfusion and protects the heart from injury. These findings have clinical implications for patients with the low-expression MIF allele.
\end{abstract}

\section{Introduction}

Macrophage migration inhibitory factor (MIF) is a proinflammatory cytokine that is released from immune cells (1) and is involved in inflammatory diseases, including rheumatoid arthritis (1), atherosclerosis (2), and sepsis (3). MIF is released from preformed storage pools and regulates the release of other inflammatory cytokines, such as tumor necrosis factor- $\alpha$ and interleukin 6 (4). MIF also counterregulates the antiinflammatory effects of glucocorticoids (5).

MIF concentration is increased in the plasma of patients with myocardial infarction (6), and ischemia increases MIF expression in rat hearts (7). Excessive MIF can reduce cardiac contractility in isolated, perfused rat hearts (8). However, MIF also regulates metabolism and increases glycolysis and glucose utilization in skeletal muscle (9). Our recent findings indicate that MIF stimulates heart muscle glucose uptake and that the autocrine/paracrine effects of endogenous cardiac MIF contribute to AMPK activation and glucose uptake during ischemia (10). AMPK has emerged as a cardioprotective stress kinase (11), and the action of MIF to modulate AMPK might be beneficial during ischemia. These findings hold clinical interest, because human cells with a low-MIF-expression allele have impaired activation of AMPK during hypoxia that is corrected by exogenous $\operatorname{MIF}(1,10)$.

JNK is a MAPK that modulates multiple cellular functions, including proliferation, differentiation, and apoptosis (12). JNK is activated by cell stress, such as heat shock and ultraviolet irradia-

Conflict of interest: Yale University has applied for a patent describing the use of MIF genotyping and MIF-directed therapies in cardiac ischemia injury.

Citation for this article: J. Clin. Invest. 119:3807-3816 (2009). doi:10.1172/JCI39738. tion (12). JNK is also activated by ischemia/reperfusion but not during ischemia alone (13). This activation is primarily mediated by the JNK upstream enzyme, MAPK kinase 4 (MKK4) (14). JNK plays a key role in regulating reperfusion injury, and JNK-knockout mouse hearts have significantly less necrosis and apoptosis induced by ischemia/reperfusion than hearts of control mice (15). Although the mechanisms responsible for JNK-regulated cell death have not been fully elucidated, previous studies indicate that activated JNK regulates proapoptotic factors such as $\mathrm{Bcl} 2$ family proteins during ischemia/reperfusion $(12,15)$.

MIF appears to regulate JNK activation in isolated cells, variably inhibiting $(16,17)$ or activating JNK $(18,19)$, depending on the cell type and conditions. These effects of MIF on JNK activation were paralleled by suppression $(16,17)$ or stimulation $(18,19)$ of apoptosis. How these cellular effects of MIF relate to its action in intact organs remains uncertain but has relevance to understanding injury during ischemia/reperfusion. We hypothesized that endogenous cardiac MIF might inhibit JNK activation during post-ischemia/reperfusion and limit injury. Therefore, we assessed the effects of genetic MIF deficiency in Mif/- hearts on JNK pathway activation during ischemia/reperfusion. We also examined whether repletion of MIF during reperfusion or inhibition of JNK activation in $\mathrm{Mif}^{\prime-}$ - hearts would improve recovery of cardiac function and decrease injury during ischemia/reperfusion.

\section{Results}

Endogenous MIF regulates JNK activation through the CD74 receptor during ischemia/reperfusion. Isolated, perfused WT and Mif/- hearts were subjected to global ischemia for 15 minutes, followed by up to 30 minutes of reperfusion, in order to determine the role of 
endogenous cardiac MIF in modulating JNK activation. In initial studies, we observed that MIF was released from WT hearts into the perfusate during reperfusion, reducing heart MIF content (Figure 1A), as previously described (10). Ischemia/reperfusion augmented the phosphorylation of JNK and downstream c-Jun in both WT and Mif/- hearts, but Mif/- hearts demonstrated a substantially greater (2.5-fold greater) JNK activation compared with WT hearts (Figure 1, A and B).

During post-ischemia/reperfusion, the deleterious effects of JNK (15) are partially balanced by activation of the prosurvival Akt pathway (20). JNK can also promote Akt activation in isolated cardiomyocytes during hypoxia-reoxygenation (21). In order to evaluate whether excessive JNK activation increased Akt activation in Mif/hearts, we assessed Akt phosphorylation after ischemia/reperfusion. However, we found that Akt phosphorylation at both the Ser473 and Thr308 sites was similar in WT and Mif/- hearts (Figure 1C).

The MIF cell-surface receptor CD74 mediates the effect of MIF to activate AMPK during hypoxia (10). To define whether CD74 is required for endogenous MIF to restrain JNK activation during reperfusion, we used hearts from $C d 74^{-/-}$syngeneic mice (BALB/c mice) (22). In initial studies, we observed normal baseline LV contractile function and histology in $C d 74^{-/-}$hearts (Supplemental Figure 1; supplemental material available online with this article; doi:10.1172/JCI39738DS1). However, during global ischemia/ reperfusion, JNK phosphorylation in $C d 74^{-/-}$hearts was comparable to that in Mif $^{/-}$hearts and was significantly higher than that in WT hearts (Figure 1D).

MIF replacement during reperfusion decreases JNK activation, contractile dysfunction, and necrosis in Mif/- hearts. We previously observed impaired recovery of contractile function during post-ischemia/ reperfusion in Mif/- hearts (10). This contractile dysfunction was associated with reduced AMPK activation during ischemia, suggesting that MIF deficiency impairs an adaptive response during the ischemic period (10). In order to determine the extent to which the lack of MIF during reperfusion contributes to excess JNK activation and greater LV dysfunction, we perfused Mif/- hearts with or without the addition of $50 \mathrm{ng} / \mathrm{ml}$ recombinant MIF (rMIF) after global ischemia. Inclusion of rMIF in the perfusate eliminated the excess JNK phosphorylation in reperfused Mif/- hearts (Figure 1E). In addition, Mif/- hearts reperfused with rMIF had significantly better recovery of LV contractile function and attenuated myocardial necrosis compared with those reperfused without rMIF (Figure $1, F$ and $G$ ). These data indicate that endogenous MIF inhibits JNK activation during reperfusion and suggest that excess JNK activation might contribute to the greater injury of Mif/- hearts during ischemia/reperfusion.

The effects of MIF on cardiac function are complex, and excessive MIF has been shown to be cardiodepressant in the heart (23). When WT hearts were perfused with $50 \mathrm{ng} / \mathrm{ml} \mathrm{rMIF}$ during reperfusion, we observed decreased contractile function during reperfusion after ischemia (Supplemental Figure 2). These findings contrast with those in the Mif/- hearts and indicate that while endogenous MIF has a protective physiologic role, excessive MIF has a detrimental effect on the recovery of function in the reperfused heart.

$M I F$ regulates $M K K 4$ during post-ischemia/reperfusion. JNK is activated by phosphorylation of upstream MKKs and MKK kinases (MKKKs) (12). Both MKK4 and MKK7 phosphorylate JNK (12), but the exact MKK isoform responsible for JNK activation in the heart during post-ischemia/reperfusion is not well understood. We observed increased phosphorylation of MKK4 but not MKK7 in reperfused hearts (Figure 2, A and B), indicating a more important role for the MKK4 isoform. In addition, Mif ${ }^{\prime-}$ hearts had greater MKK4 activation compared with WT hearts (Figure 2A). Furthermore, reperfusion of Mif/- hearts with $50 \mathrm{ng} / \mathrm{ml} \mathrm{rMIF}$ following ischemia eliminated their excess MKK4 activation (Figure 2C). Taken together, these results indicate that endogenous MIF prevents excess MKK4 activation during reperfusion.

Several MKKKs regulate MKK4, including apoptosis signal-regulating kinase 1 (ASK1), transforming growth factor- $\beta$-activating kinase 1 (TAK1), and mixed lineage kinases (MLKs) (12). In order to better understand the mechanism responsible for the excess MKK4 activation in the Mif/- hearts, we evaluated the activation of these kinases in Mif/- and WT hearts. Mif/- and WT hearts had similar increases in phosphorylation at the major ASK1 inhibitory site Ser967, suggesting that ASK1 is not responsible for increased MKK4/JNK activation (Figure 2D). TAK1 Thr184/187 phosphorylation did not change with ischemia/reperfusion (data not shown). MLK3 phosphorylation on its Thr277/Ser281-activating sites increased to a similar degree in WT and Mif/- hearts (Figure 2E). These results suggest that MIF is either affecting other regulatory sites, or alternatively inhibiting MKK4 phosphorylation or promoting MKK4 dephosphorylation.

ER stress has been reported to increase JNK activation (24), presumably through upstream kinases such as MKK4. Thus, we assessed whether ER stress markers were altered in Mif ${ }^{-1}$ hearts. However, there were no differences in either the phosphorylation of protein kinase RNA-like ER kinase (PERK) or the expression of ER chaperone (Bip) between Mif/- and WT hearts during ischemia/reperfusion (Supplemental Figure 3).

Excess JNK activation in Mif/- hearts leads to contractile dysfunction and altered BCL2-associated agonist of cell death phosphorylation. JNK activation is known to promote cardiac injury following ischemia/reperfusion (15). In order to determine whether there might be a causal relationship between JNK activation and contractile dysfunction in Mif $^{\prime-}$ hearts following ischemia, we perfused hearts with the JNK inhibitor, SP600125, which blocked excessive JNK activation during reperfusion (Figure 3A). SP600125 significantly improved cardiac function during post-ischemia/reperfusion in Mif ${ }^{-}$- hearts (Figure $3 \mathrm{~B}$ ), indicating that excessive JNK activation in Mif/- hearts contributes to poor recovery of contractile function after ischemia.

JNK mediates cardiac injury, in part, through the induction of apoptosis during ischemia/reperfusion $(15,25)$. JNK reduces BCL2-associated agonist of cell death (BAD) phosphorylation at the Ser112 site that mediates its interaction with antiapoptotic factors (15). BAD Ser112 phosphorylation decreased in both WT and Mif $^{\prime-}$ hearts following ischemia/reperfusion. However, Mif ${ }^{\prime-}$ hearts demonstrated less phosphorylation compared with WT hearts, which would represent a proapoptotic effect (Figure 3C). This effect was reversed by treatment with SP600125 (Figure 3C).

The effects of MIF on JNK phosphorylation in H9C2 cardiomyocytes. MIF is secreted during ischemia/reperfusion from preformed pools and exerts autocrine-paracrine effects in the heart (10). To assess the autocrine role of MIF in JNK activation in an isolated cell model, we subjected H9C2 cardiomyocytes to hypoxia-reoxygenation in the presence of either neutralizing anti-MIF antibody or nonimmune IgG. After hypoxia-reoxygenation, H9C2 cells had increased JNK phosphorylation (Figure 4A). Immunoneutralization of MIF accentuated JNK activation (Figure 4A), demonstrating that endogenous, secreted MIF has an autocrine effect to suppress JNK activation. 
A
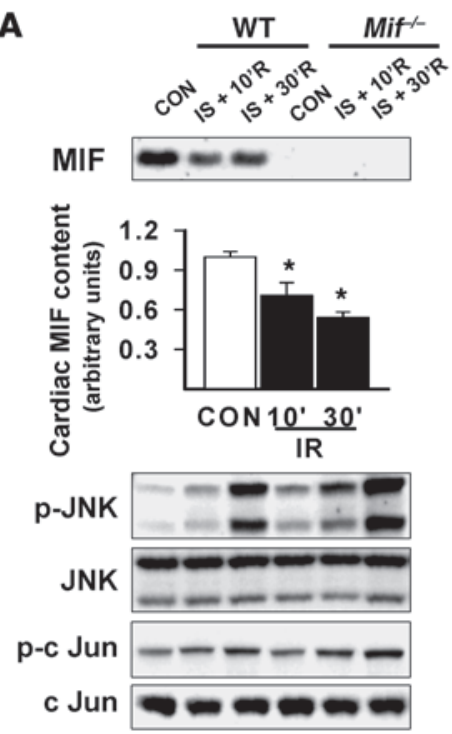

D

D $\prod_{\text {CON IR CON IR CON IR }}^{\text {WT }}$
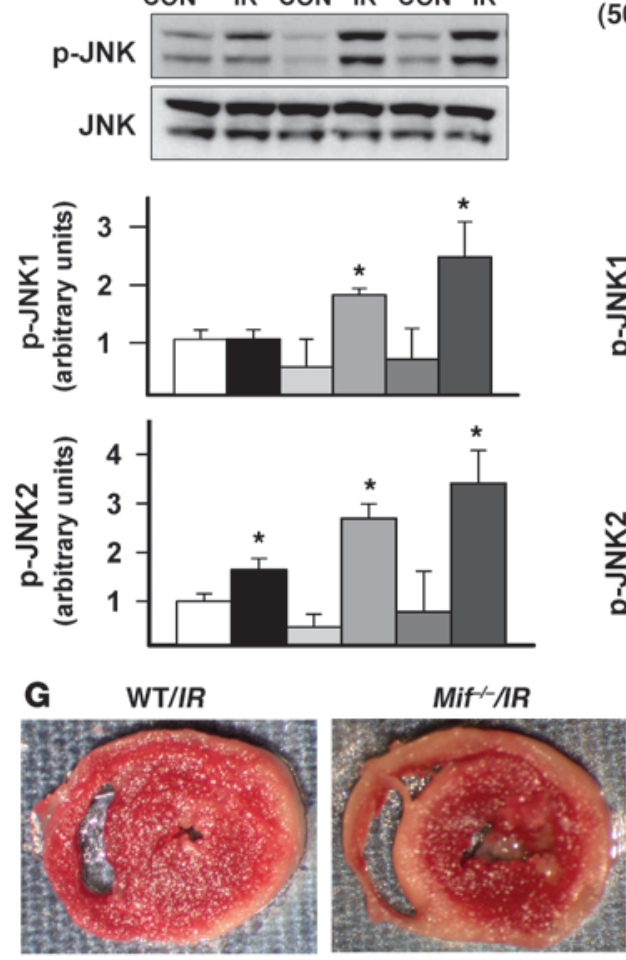

B
p-JNK
JNK
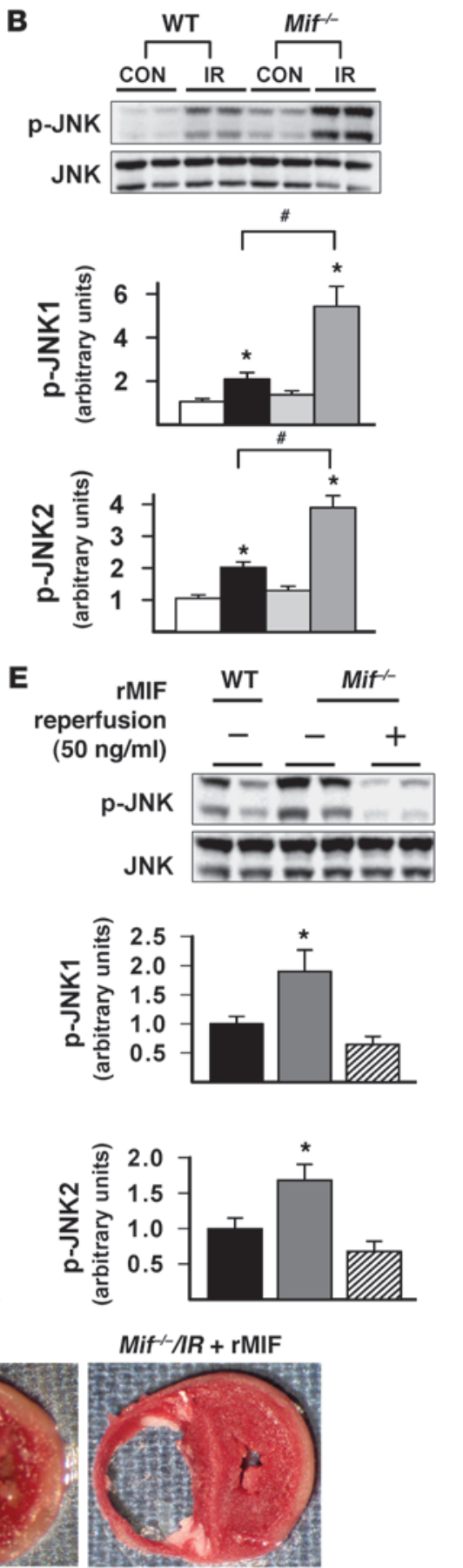

C
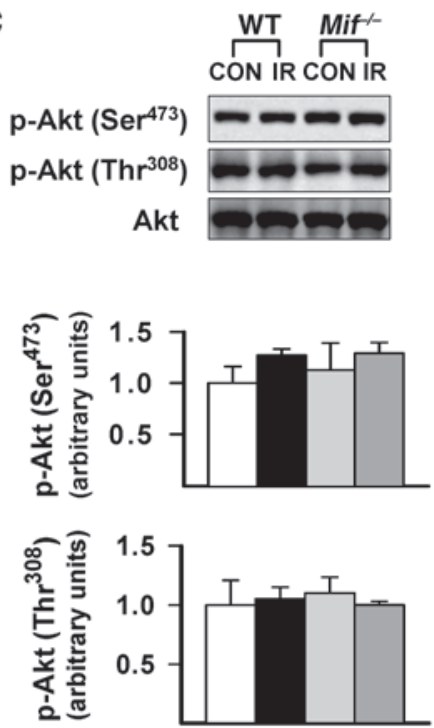

$\mathbf{F}$
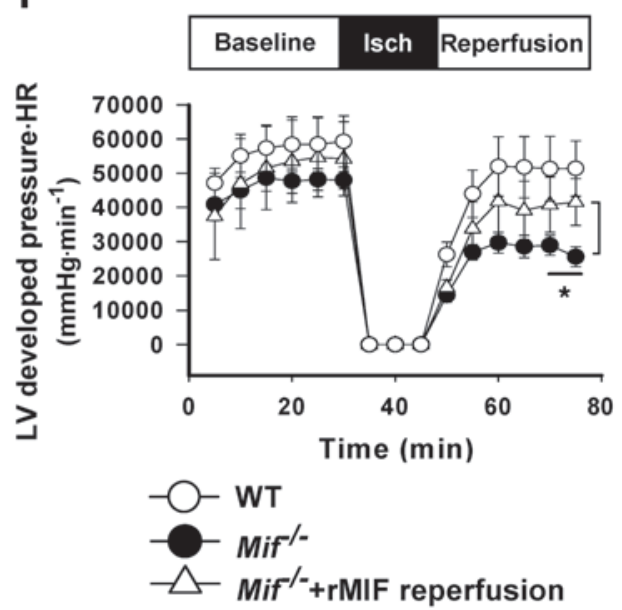

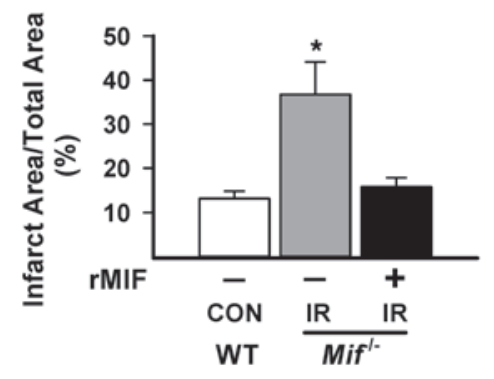

Figure 1

Endogenous MIF modulates JNK activation, heart function, and cardiac injury following global ischemia/reperfusion in vitro. Isolated, perfused hearts from WT, $\mathrm{Mif}^{-1}$, and $\mathrm{Cd} 74^{-/-}$mice underwent control (CON) perfusion and 15 minutes of no-flow ischemia (IS) with reperfusion. (A) Hearts underwent no-flow ischemia with reperfusion for 10 minutes (10'R) or 30 minutes (30'R), and cardiac MIF content was assessed by immunoblotting. (B-D) Hearts underwent no-flow ischemia with reperfusion for 30 minutes (IR), and JNK, Akt, and C-Jun immunoblots were performed on heart homogenates with phospho-specific and total antibodies. (E-G) Additional Mif/- hearts were subjected to no-flow ischemia with reperfusion, with or without addition of $\mathrm{rMIF}(50 \mathrm{ng} / \mathrm{ml})$ in the perfusate during reperfusion.

(E) Immunoblots were performed on heart homogenates with phospho-specific and total antibodies. (F) LV contractile function was assessed as heart rate (HR) times LV systolic pressure. isch, ischemia. (G) Hearts were stained with TTC to define the infarct area. Quantification of immunoblots is shown in the graphs below the blots. $n=3-6$ per group; ${ }^{*} P<0.05$ versus control; ${ }^{\#} P<0.05$ versus WT. 
A
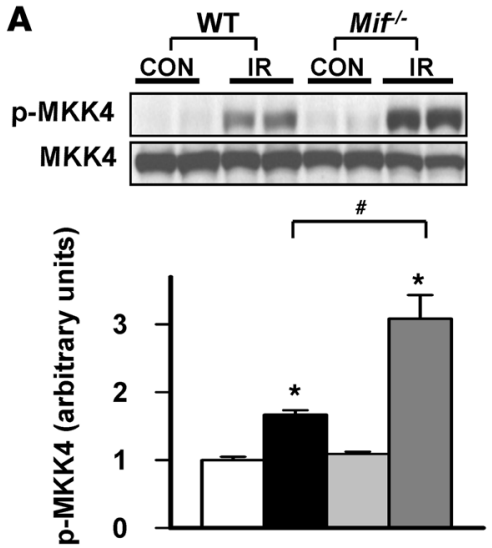

D
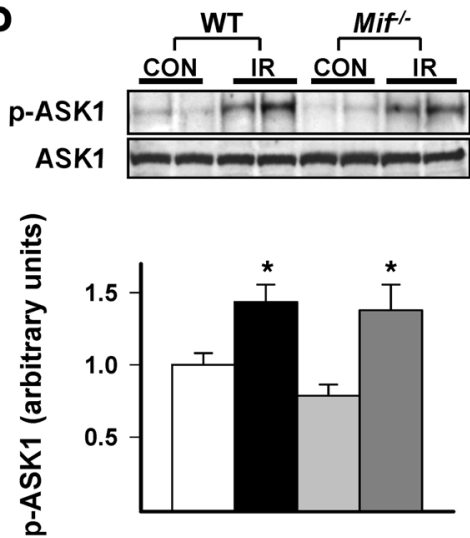

B
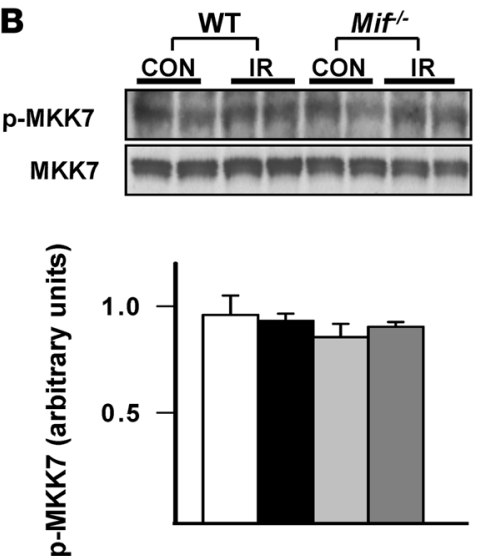

$\mathbf{E}$
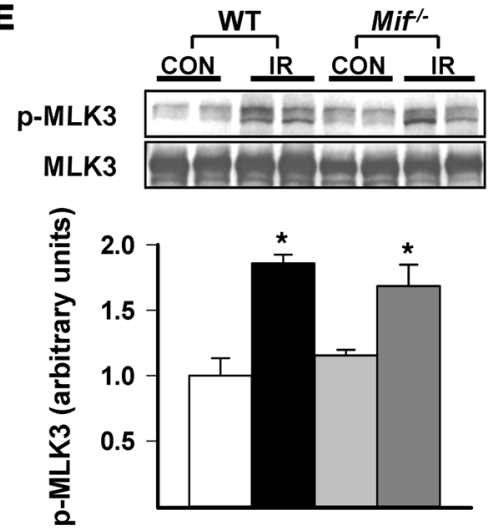
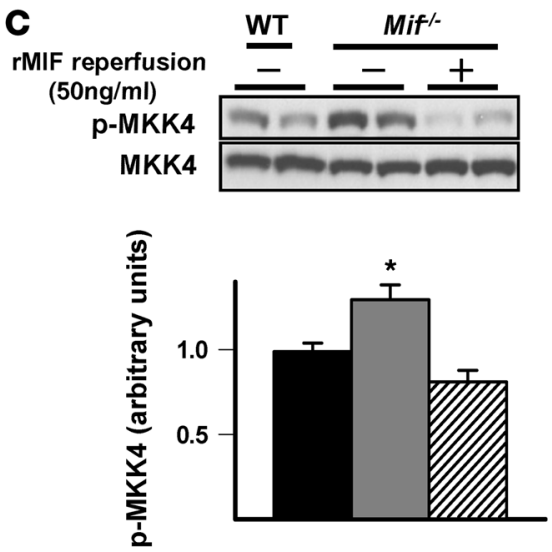

Figure 2

Regulation of heart MKKs and MKKKs by endogenous MIF following ischemia/reperfusion in vitro. Isolated, perfused hearts from WT and Mif ${ }^{-1-}$ mice underwent control perfusion or 15 minutes of no-flow ischemia with reperfusion. Heart homogenates were immunoblotted with phospho-specific and/or total antibodies to (A) MKK4, (B) MKK7, (D) ASK1, and (E) MLK3. (C) Additional Mif /- $^{-}$hearts were reperfused with or without rMIF (50 ng/ml) and immunoblotted with phospho-specific and total MKK4 antibodies. Quantification of immunoblots is shown in the graphs below the blots. $n=4$ per group; ${ }^{*} P<0.05$ versus control; $\# P<0.05$ versus WT.

In order to determine whether MIF suppresses JNK activation through stimuli other than hypoxia, we treated H9C2 cells with anisomycin, a potent activator of JNK (26). Anisomycin increased JNK phosphorylation in a dose-dependent manner (Supplemental Figure 4). However, incubation with $\mathrm{rMIF}(50 \mathrm{ng} / \mathrm{ml})$ inhibited anisomycin-stimulated JNK and c-Jun phosphorylation (Figure 4, $B$ and C). These results indicate that MIF has a short-term effect to inhibit JNK activation under nonhypoxic conditions.

Endogenous MIF blunts JNK activation and injury following ischemia/ reperfusion in vivo. Patients with myocardial infarction have elevated plasma MIF concentrations (6). In vivo, the combined effects of cardiac and noncardiac MIF might impact on the physiologic response of the heart to ischemia/reperfusion. Thus, to test whether whole-body MIF deficiency leads to excess heart JNK activation in vivo, we subjected WT and Mif/- mice to left coronary artery occlusion for 20 minutes, followed by 3 hours of reperfusion. In the WT mice, plasma MIF had a 3-fold increase during ischemia/ reperfusion (Figure 5A). The heart MIF content also decreased by $30 \%$, suggesting that cardiac MIF release contributes to the increase in plasma MIF observed (Figure 5B).

Similar to our observations in the isolated, perfused heart, coronary artery occlusion and reperfusion increased JNK activation to a greater extent in Mif/- than in WT mice in the ischemic region
(Figure 5C). Proapoptotic JNK-mediated BAD dephosphorylation was also accentuated in Mif/- mice compared with WT mice in vivo (Figure $5 \mathrm{C}$ ). Mif/- hearts also demonstrated more cleaved poly(ADP-ribose) polymerase (PARP) protein on Western blots and apoptotic cells through TUNEL staining when compared with WT hearts (Figure 5D). To more directly assess the role of JNK pathway activation in mediating injury in the Mif/- mice, we pretreated mice with the JNK inhibitor SP600125 or vehicle prior to coronary occlusion, as previously described (27). SP600125 treatment effectively decreased JNK phosphorylation (Supplemental Figure 5) and cell apoptosis (Figure 5D), as compared with those in the control group. Mif/- mice had more necrosis than WT mice, based on analysis of triphenyl tetrazolium chloride-stained (TTC-stained) LV sections and higher serum troponin I concentrations after ischemia/reperfusion (Figure 5, E and F). However, pretreatment with SP600125 reduced both parameters of necrosis in Mif/- mice (Figure 5, E and F). Thus, these results indicate that excess JNK activation contributes to myocardial injury during ischemia/reperfusion in vivo.

Recent studies have linked AMPK activation to inhibition of JNK activation in endothelial cells (28). Since we have previously found that AMPK activation is blunted in Mif/- hearts during ischemia (10), we tested whether impaired AMPK activity leads to increased JNK activation, using the well-characterized AMPK kinase dead 
A

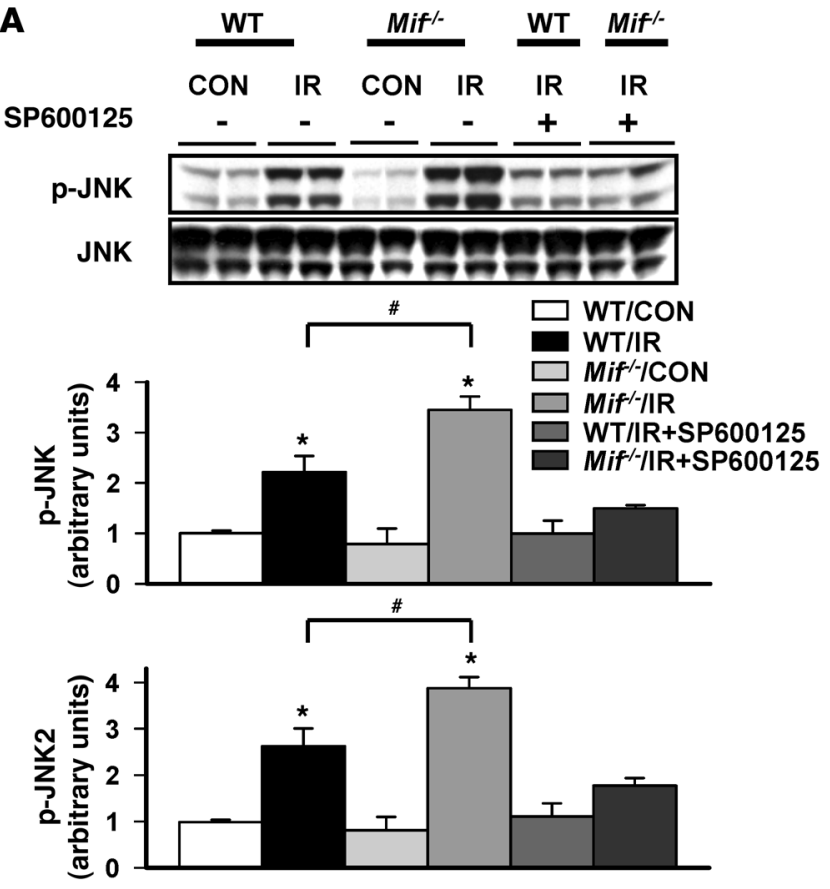

B

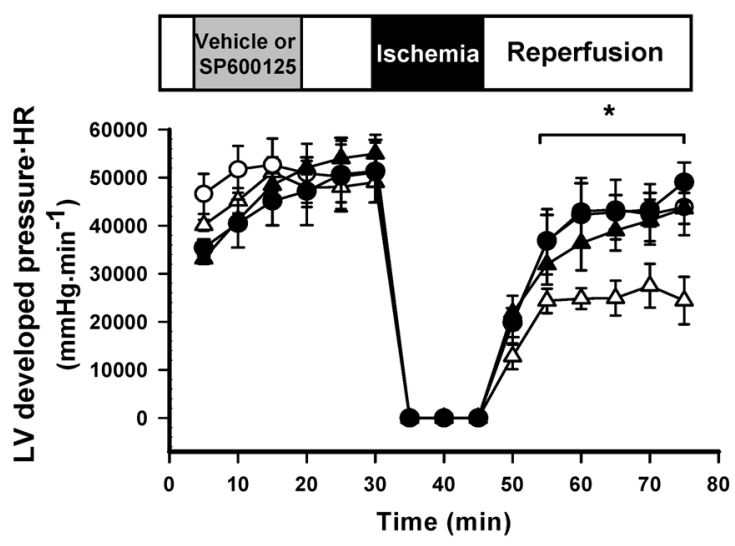

- WT/IR
- WT/IR+SP600125
$-\triangle M i f^{/-I R R}$
$\triangle M i f^{/-I R+S P 600125}$
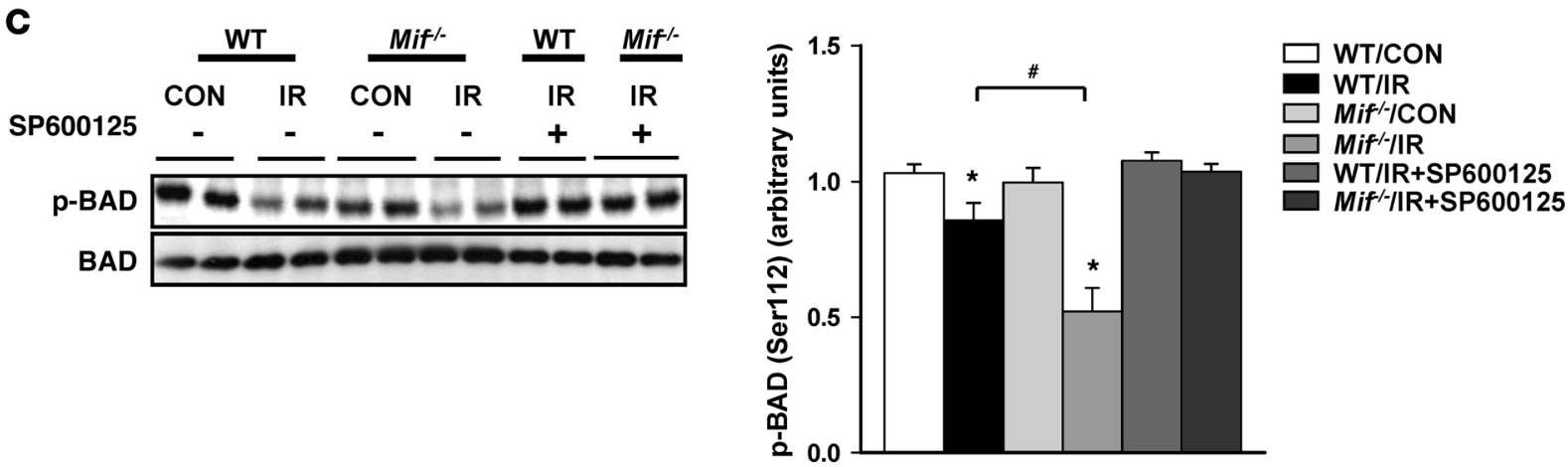

Figure 3

Effects of JNK inhibition on heart function and BAD phosphorylation following ischemia/reperfusion in vitro. Hearts from WT and Mif ${ }^{-1}$ mice underwent control perfusion or 15 minutes of no-flow ischemia and 30 minutes of reperfusion, with or without SP600125 (10 umol/I). Heart homogenates were immunoblotted with phospho-specific or total antibodies to (A) JNK and (C) BAD. Quantification of immunoblots is shown in the graphs. (B) LV contractile function was assessed as heart rate times LV systolic pressure. $n=4-6$ per group; ${ }^{*} P<0.05$ versus control; $\# P<0.05$ versus WT.

(KD) mouse model (11). We found that heart JNK phosphorylation was greater in AMPK KD mice compared with WT mice following coronary artery occlusion-reperfusion (Supplemental Figure 6), suggesting that AMPK plays a role in modulating JNK activation during reperfusion.

Polymorphismsin the human MIFpromoterinfluence cellularJNKactivation. A functional polymorphism in the human MIF gene promoter modulates MIF expression. The promoter contains either 5, 6, 7, or 8 CATT tetra-nucleotide repeats at position -794 , and the CATT 5 allele demonstrates the lowest level of MIF promoter activity when compared with the others $(1,10)$. In order to determine whether this genetic difference influences JNK activation, we studied human fibroblasts homozygous for the $\mathrm{CATT}_{5}$ allele $(5 / 5$ cell lines) and others with at least 1 high expression 6- or 7-CATT allele (non$5 / 5$ cell lines). As anticipated, following hypoxia-reoxygenation, the MIF concentration in the incubation media increased least in the
5/5 cells (Supplemental Figure 7). Hypoxia-reoxygenation stimulated JNK activation and downstream c-Jun phosphorylation in all cells; however, the low-MIF-expression 5/5 cells showed the greatest level of JNK pathway activation (Figure 6). These results suggest that the activation of JNK during ischemia/reperfusion might vary in patients, depending on their MIF genotype.

\section{Discussion}

MIF is a proinflammatory cytokine, which plays an important role in chronic inflammatory diseases (1). However, MIF is also expressed in several tissues, including the heart, in which it has a physiologic role during ischemia (29). The current results indicate that endogenous cardiac MIF inhibits activation of the JNK pathway and limits myocardial reperfusion injury after ischemia. Specifically, hearts from $\mathrm{Mif}^{\prime-}$ mice had increased activation of the MKK4/JNK/c-Jun pathway during reperfusion. Excess activa- 
A

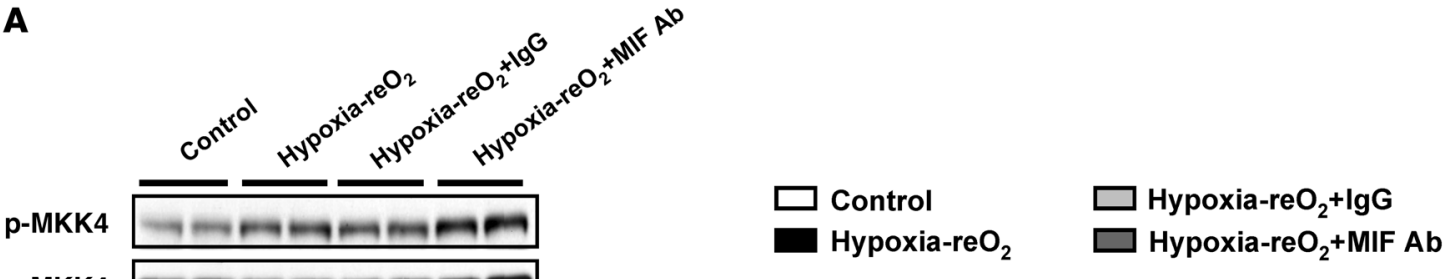

MKK4

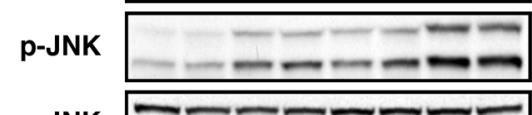

JNK

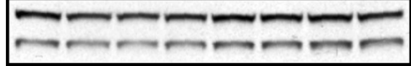

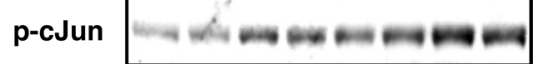

cJun

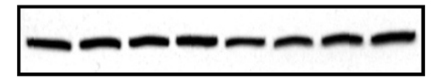

B

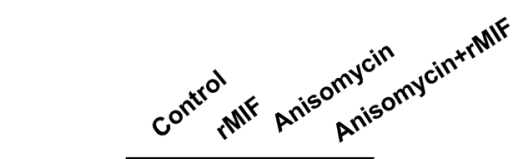

p-MKK4

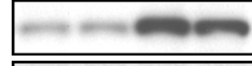

MKK4

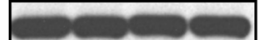

p-JNK

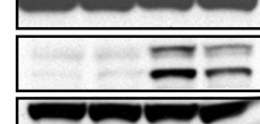

JNK
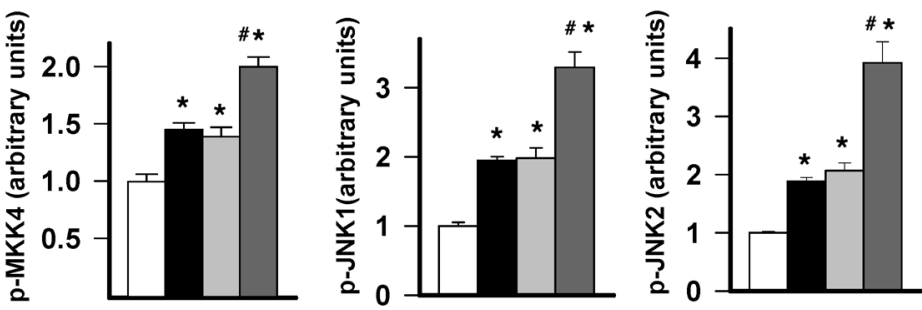

C

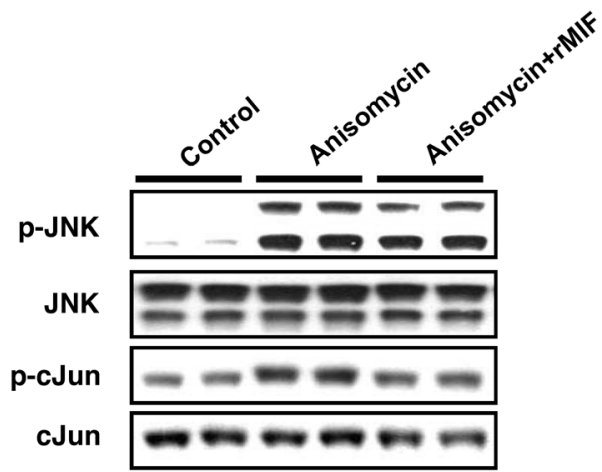

$\square$ Control $\square$ Anisomycin+rMIF

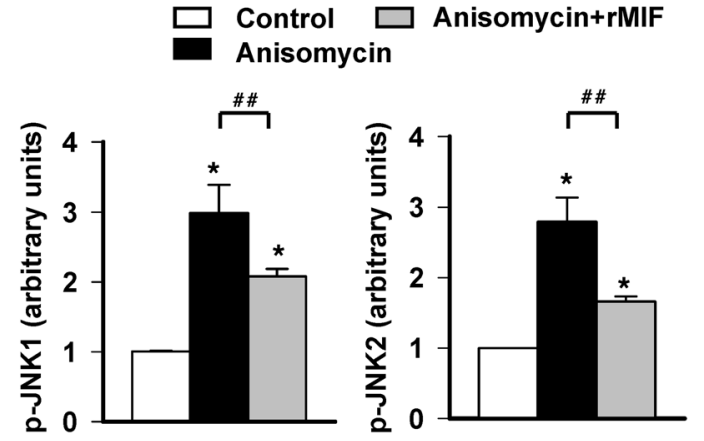

Figure 4

MIF regulates JNK activation during hypoxia-reoxygenation and anisomycin treatment. (A) H9C2 cardiomyocyte cells were subjected to normoxic incubation (control) or 1 hour of hypoxia and 30 minutes of reoxygenation $\left(\mathrm{Hypoxia}_{-} \mathrm{reO}_{2}\right)$. Cells were incubated alone $\left(\mathrm{Hypoxia} \mathrm{reO} \mathrm{O}_{2}\right)$, with nonspecific IgG (Hypoxia-reO ${ }_{2}+\mathrm{lgG}$ ), or MIF-specific antibody (Hypoxia-reO ${ }_{2}+\mathrm{MIF} \mathrm{Ab}$ ) to neutralize secreted endogenous MIF. MKK4 and JNK were immunoblotted with phospho-specific and total antibodies. (B and C) H9C2 cells were treated with anisomycin $(0.01 \mu \mathrm{g} / \mathrm{ml})$, with or without the addition of exogenous rMIF (50 ng/ml) for 1 hour. JNK and c-Jun were immunoblotted with phospho-specific and total antibodies. Quantification of immunoblots is shown in the graphs. $n=4$ per group; ${ }^{*} P<0.05$ versus control; ${ }^{\#} P<0.05$ versus 1 hour of hypoxia and 30 minutes of reoxygenation groups; $\# P<0.05$ versus anisomycin.

tion of this pathway promoted greater BAD dephosphorylation and increased both apoptosis and necrosis in Mif/- hearts. MIF deficiency during the reperfusion period appeared to have an important role in the development of injury, since MIF replacement during reperfusion improved recovery of cardiac function and reduced necrosis. These mechanisms also were operative in Mif/- mice during in vivo coronary occlusion and reperfusion. Finally, we found that human cells with a low-MIF-expression genotype had accentuated JNK activation after hypoxia-reoxygen- ation. These latter findings have potential relevance to patients with the low-MIF-expression genotype who might be more susceptible to ischemic injury.

JNK is activated by various types of cell stress, including oxidative stress, UV irradiation, heat shock, and ischemia/reperfusion (12). Our results demonstrate that endogenous cardiac MIF suppresses the activation of the JNK pathway through the action of its receptor, CD74, during ischemia/reperfusion. Whether this observation reflects autocrine or paracrine effects of MIF, or both, in the heart is 
A

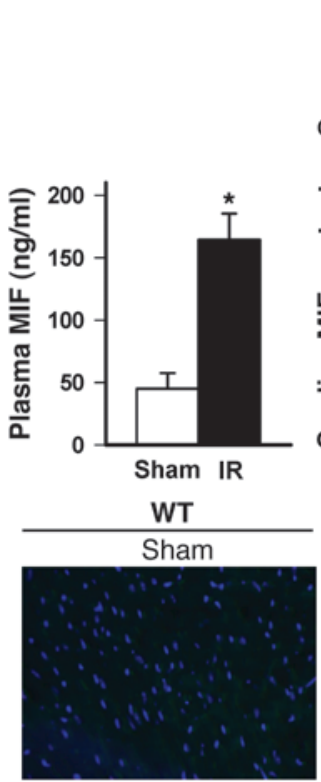

IR

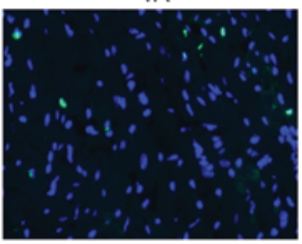

$\mathrm{IR}+\mathrm{SP}$

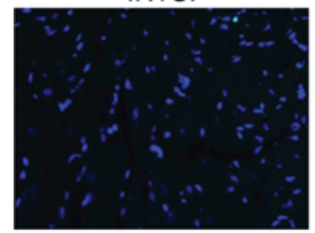

E

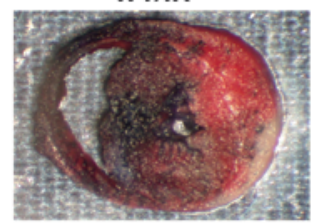

WT/IR+SP

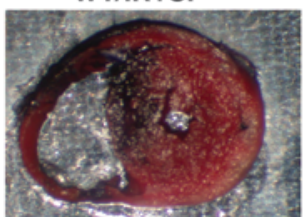

B
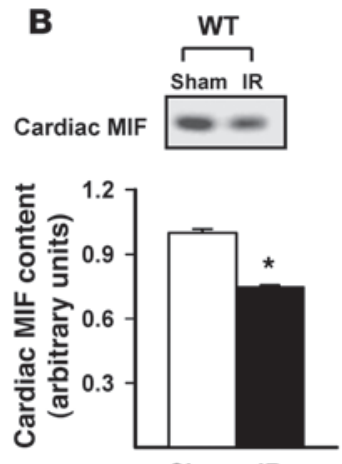

Sham IR

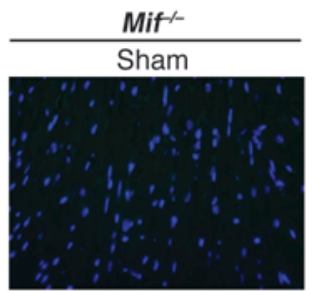

IR

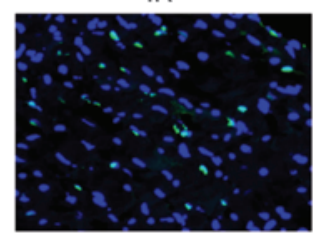

$\mathrm{IR}+\mathrm{SP}$

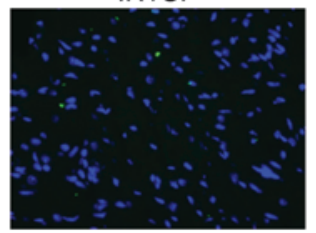

Mif 1 -IIR

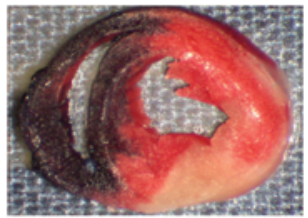

Mif $^{\prime-/ I R+S P}$

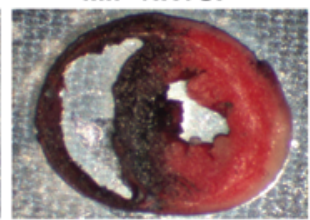

C
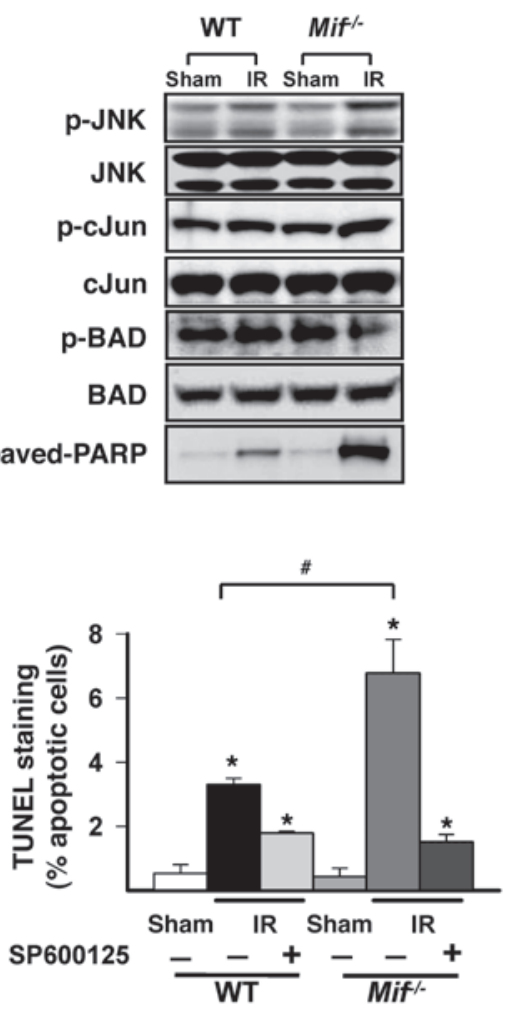

Figure 5

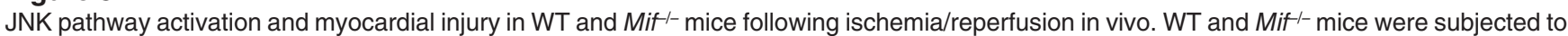
sham thoracotomy (sham) or 20 minutes of left coronary artery ligation, followed by 3 hours of reperfusion. (A) Plasma MIF concentration after reperfusion was measured by ELISA. (B) The cardiac content of MIF was assessed by immunoblots. Quantification of the immunoblot is shown in the graphs below the blot. (C) JNK, c-Jun, cleaved PARP, and Bad were assessed in homogenates from the ischemia/reperfusion region by immunoblotting with phospho-specific and total antibodies. (D) WT and Mif/- mice were pretreated with SP600125 (SP) (1 mg/kg) (ischemia/ reperfusion plus SP600125) or vehicle (ischemia/reperfusion) intravenously, 15 minutes prior to coronary occlusion-reperfusion. Hearts were fixed and apoptotic nuclei were identified by TUNEL staining (original magnification, $\times 40$ ). Additional hearts were dual stained with TTC and blue dye to identify viable myocardium versus necrosis within the ischemic region. (E) The ischemic risk area was expressed as percentage of the heart; infarct size was expressed as percentage of ischemic risk area. (F) Plasma troponin I was measured at the end of control or ischemia/ reperfusion experiments. $n=4$ per group; ${ }^{*} P<0.05$ versus sham; ${ }^{*} P<0.05$ versus WT. 
A

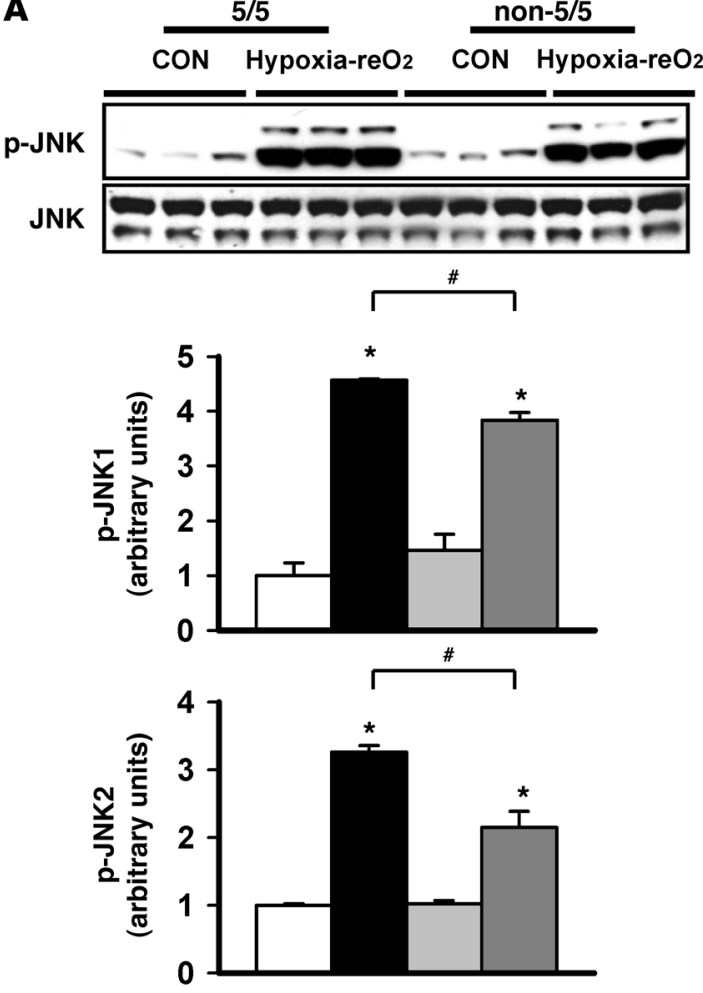

B
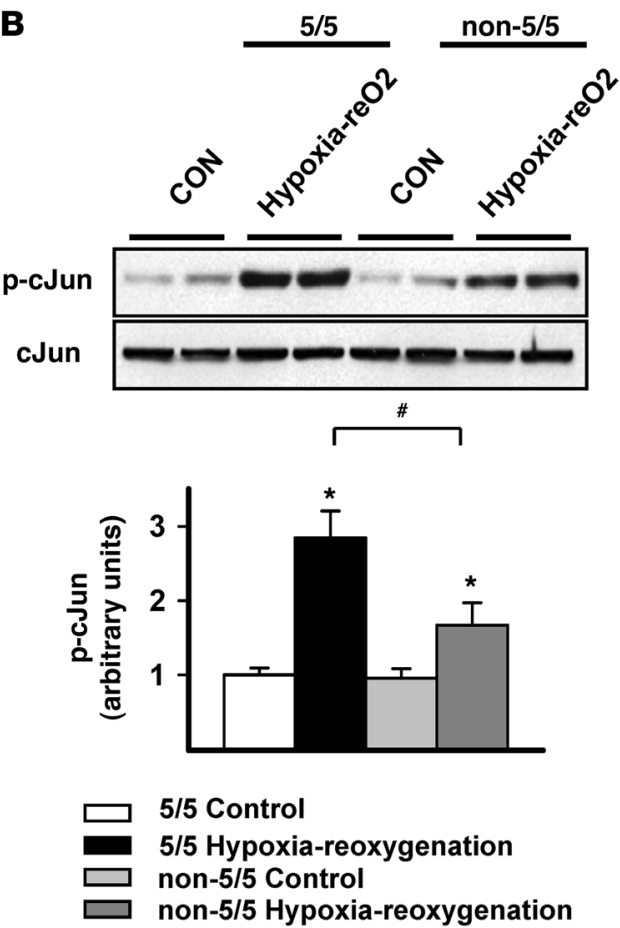

Figure 6

JNK activation following hypoxia-reoxygenation according to MIF promoter genotype in human fibroblasts. Dermal fibroblasts from subjects with the MIF promoter low-expression 5-CATT repeat allele $(5 / 5$ genotype; $n=5)$ and subjects with at least 1 high expression 6 - or 7 -CATT repeat allele (non-5/5 genotype; $n=7$ ) were subjected to normoxic control incubation or 9 hours of hypoxia and 30 minutes of reoxygenation (hypoxia$\mathrm{reO}_{2}$ ). Lysates were immunoblotted with phospho-specific and total JNK $(\mathbf{A})$ and c-Jun (B) antibodies. Quantification of immunoblots is shown in the graphs below the blots. ${ }^{*} P<0.05$ versus control; $\# P<0.05$ versus $5 / 5$ hypoxia-reoxygenation.

uncertain. However, the former possibility is suggested by our finding that MIF has an autocrine action to suppress JNK pathway activation in heart-derived $\mathrm{H} 9 \mathrm{C} 2$ cells during hypoxia-reoxygenation. Suppression of the JNK pathway by MIF is consistent with previous observations that MIF inhibits JNK activation in HeLa cells (16) and reduces apoptosis in noncardiac cells (17). In contrast, MIF stimulates the JNK pathway in other cells, leading to the expression of matrix metalloproteinases $(18,30)$. The duration of MIF exposure might be important in determining its effect. Prolonged incubation with MIF appears to increase JNK phosphorylation and cell apoptosis in neonatal cardiomyocytes (19). LDL cholesterol increases MIF expression and JNK activation in human mesangial cells (31). Thus, the relationship between MIF and the JNK pathway may depend on the duration of exposure and the cell type.

JNK is activated by 2 upstream kinases, MKK4 and MKK7 (12). Our results and others indicate that MKK4 is the primary upstream JNK kinase, which is activated during ischemia/reperfusion in the heart $(14,32)$. MKK7 is activated by ischemia/reperfusion in brain (33), but we did not observe this in the heart. However, the absence of endogenous cardiac MIF during reperfusion led to increased MKK4 activation, which likely is responsible for the excess activation of the JNK pathway. The MKK4 upstream kinases (MKKKs) include MLK3 $(34,35)$, ASK1 (36), and TAK1 (37). Only MLK3 phosphorylation increased during ischemia/reperfusion in our experiments; however, it did so to a similar extent in WT and
Mif/- hearts. Thus, these results suggest that MIF either affects other MKKKs or MKKK regulatory sites, or potentially modulates MKK4 dephosphorylation by protein phosphatases.

The protective effect of exogenous rMIF during reperfusion in Mif $^{\prime \prime}$ - hearts was likely due to its suppression of excessive JNK pathway activation. We found that JNK pathway inhibition improved the postischemic recovery of contractile function in Mif/- hearts and reduced myocardial necrosis in Mif/- mice after coronary artery ligation-reperfusion. Consistent with our results, JNK-knockout mice are known to have less myocardial injury following ischemia/reperfusion (15). However, MKK7-overexpressing mice have increased JNK activation but are also less susceptible to ischemic injury (15). This paradoxical finding might be explained in part by their abnormal baseline phenotype (hypertrophy and increased contractility).

Our results also indicate that enhanced JNK activation is responsible for the increased apoptosis in Mif/- hearts during ischemia/ reperfusion in vivo. These observations are consistent with findings in isolated adult cardiomyocytes, in which activation of JNK by MKK4 triggers the mitochondrial apoptosis pathway (25). JNK activation also is known to induce apoptosis in other tissues (15, 38). However, the relationship between JNK activation and apoptosis varies depending on the cell type and stimulus. For instance, neonatal cardiomyocytes have reduced apoptosis during hypoxia-reoxygenation, when treated with JNK inhibitors (21). Nonetheless, in the 
intact heart, JNK activation appears to inhibit BAD Ser112 phosphorylation (15), which decreases its interaction with antiapoptotic factors. Previous results have shown that $J n k^{-/-}$mice had increased BAD Ser112 phosphorylation (15), consistent with our finding that excessive JNK activation was associated with reduced BAD Ser112 phosphorylation in Mif/- hearts during ischemia/reperfusion.

The current findings complement our recent observation that MIF deficiency compromises the physiologic response during ischemia by blunting heart AMPK activation (10). Taken together, they indicate that endogenous MIF has dual cardioprotective effects, amplifying the AMPK response during ischemia and blunting the JNK response during reperfusion. Interestingly, a recent study in endothelial cells found that pharmacologic AMPK stimulation attenuated JNK activation during exposure to hydrogen peroxide (28). In support of a possible link between AMPK and JNK signaling in the heart, we observed greater JNK activation in AMPK-deficient mouse hearts compared with WT mouse hearts following ischemia/ reperfusion. These results raise the possibility that the impaired AMPK activation, which we previously reported during ischemia in Mif/- hearts (10), might either directly or indirectly (through reduction in ischemic injury) contribute to the excess JNK activation. However, the finding that MIF replacement during reperfusion significantly reduced JNK activation in Mif/- hearts highlights the important effects of endogenous MIF during reperfusion.

Our findings that MIF modulates JNK activation during ischemia/reperfusion may have relevance to human disease. The common polymorphism in the human MIF gene promoter leads to variable levels of constitutive and induced MIF expression (1). This polymorphism has been linked to the clinical manifestations of inflammatory diseases, including asthma (39), cystic fibrosis (40), and rheumatoid arthritis (1). We recently showed that the low$M I F-$ expression genotype led to reduced AMPK activation during hypoxia (10). The current findings further demonstrate that the $M I F$ genotype modulates the activation of the JNK pathway. Taken together, these observations suggest that patients with a low-MIFexpression genotype may be more susceptible to myocardial injury during ischemia/reperfusion. They also raise the possibility that JNK pathway inhibition might be a particularly effective strategy to prevent injury during acute coronary syndromes in these patients.

\section{Methods}

Experimental animals. WT (age, 10-16 weeks; Charles River Laboratories), $\mathrm{Mif}^{\prime-}, \mathrm{C} d 74^{-/-} \mathrm{BALB} / \mathrm{c}$, and AMPK KD C57BL/6 mice (generation N10, male) (39) were fed and housed according to the guidelines from the American Association for Laboratory Animal Care. All procedures were approved by the Yale University Animal Care and Use Committee.

Heart perfusions and global ischemia. Mice were anesthetized with pentobarbital sodium ( $60 \mathrm{mg} / \mathrm{kg}$ i.p.), and hearts were removed and retrogradely perfused with Krebs-Henseleit buffer (10). Hearts were stabilized at flow rate of $4 \mathrm{ml} / \mathrm{min}$ for 30 minutes, followed by either continued normal perfusion ( $4 \mathrm{ml} / \mathrm{min}$ for 45 minutes) or 15 minutes of global ischemia plus reperfusion (30 minutes), with or without $\mathrm{rMIF}(50 \mathrm{ng} / \mathrm{ml})$ prepared following the procedure of Bernhagen et al. (41). To test the effects of JNK pathway inhibition, hearts from WT and $M i f^{\prime-}$ mice were initially perfused for 10 minutes, treated with $10 \mu \mathrm{mol} / \mathrm{l} \mathrm{SP} 600125$ for 15 minutes, perfused without SP600125 for 5 minutes (42), and then subjected to 15 minutes of no-flow ischemia and 30 minutes of reperfusion.

Coronary ligation and regional ischemia. WT and Mif/- mice were anesthetized and ventilated (Rodent Ventilator, model 683; Harvard Instruments) with room air supplemented with oxygen (10). After left thoracotomy, the left coronary artery was ligated $2 \mathrm{~mm}$ below the left atrial appendage. Following 20 minutes of coronary occlusion, the ligature was released, and the heart was reperfused for 3 hours. Sham operations were performed by placement of a suture without ligation. Core temperature was maintained and the electrocardiogram was monitored to document ST-segment elevation during coronary occlusion and to detect arrhythmias.

MIF measurements. Heart perfusate and plasma MIF concentrations were measured by ELISA (10).

Western blotting. Heart homogenate proteins were resolved by SDS-PAGE and immunoblotted (10) with rabbit phospho-JNK antibody from Promega, and JNK, c-Jun, MKK4, ASK1, BAD, MLK3, phospho-c-Jun (Ser73), MKK4 (Thr261), Akt (Ser473), Akt (Thr308), ASK1 (Ser967), BAD (Ser112), MLK3 (Thr277/Ser281), and cleaved PARP antibodies from Cell Signaling.

TUNEL and TTC staining. Hearts were fixed in 10\% formalin and embedded in paraffin for TUNEL staining (Roche). Unfixed hearts were stained following in vitro and in vivo ischemia/reperfusion to delineate the extent of myocardial necrosis (10). Viable tissue in the ischemic region was stained red by TTC, leaving necrotic tissue white; the in vivo nonischemic region was stained blue with Evan's blue dye. Hearts were fixed and sectioned into 1-mm slices, photographed, and analyzed using NIH ImageJ software (http://rsbweb.nih.gov/ij/).

Troponin I. Serum troponin I was measured by ELISA (Life Diagnostics). H9C2 cells. Cardiac H9C2 cells (ATCC) were cultured in DMEM, with $10 \%$ FBS and $1 \%$ antibiotic (streptomycin and penicillin). To examine the regulation of JNK activation, the H9C2 cells were incubated with variable concentrations of anisomycin $(0.001-0.05 \mu \mathrm{g} / \mathrm{ml})$ for 1 hour. The effects of MIF on JNK activation were examined by coincubating H9C2 cells with anisomycin and $\mathrm{rMIF}(50 \mathrm{ng} / \mathrm{ml})$. To study the effects of MIF during hypoxia and reoxygenation, $\mathrm{H} 9 \mathrm{C} 2$ cells were incubated with $100 \mu \mathrm{g} / \mathrm{ml}$ of nonimmune $\mathrm{IgG}$ or a neutralizing anti-MIF antibody (10) and then were placed in a chamber filled with $95 \%$ nitrogen $/ 5 \% \mathrm{CO}_{2}$ for 1 hour, followed by room air $/ 5 \% \mathrm{CO}_{2}$ for 30 minutes. At the end of treatments, cells were lysed and immunoblotted.

Human fibroblasts. Human dermal fibroblasts, obtained from the Yale Human Cell Resource Center, were used according to the regulations and with the approval of the Yale Human Investigation Committee. Fibroblasts were cultured in DMEM medium, including 10\% FBS, penicillin $(100 \mathrm{IU} / \mathrm{ml})$, and streptomycin $(100 \mu \mathrm{g} / \mathrm{ml})$ at $37^{\circ} \mathrm{C}$. MIF genotype was determined by polymerase chain reaction (10). Early-passage fibroblasts were then incubated for either 9.5 hours of normoxia (room air $/ 5 \% \mathrm{CO}_{2}$ ) or 9 hours of hypoxia $\left(95 \%\right.$ nitrogen $\left./ 5 \% \mathrm{CO}_{2}\right)$, followed by 30 minutes of reoxygenation.

Statistics. Values are mean \pm SEM. One-way ANOVA followed by the Tukey tests or the unpaired and paired 2-tailed Student's $t$ test with Bonferroni correction were used to determine differences between group mean values. $P$ values of less than 0.05 were considered statistically significant.

\section{Acknowledgments}

This study was supported by NIH grant R01 HL-63811, NIH grant A1043210, the Brookdale Foundation, the American Heart Association, the Canadian Diabetes Association, and the Studienstiftung des deutschen Volkes.

Received for publication May 4, 2009, and accepted in revised form October 7, 2009.

Address correspondence to: Lawrence H. Young, Section of Cardiovascular Medicine, Department of Internal Medicine, Yale University School of Medicine, 333 Cedar Street, New Haven, Connecticut 06520, USA. Phone: (203) 785-4102; Fax: (203) 785-7567; E-mail: lawrence.young@yale.edu. 
1. Baugh, J.A., et al. 2002. A functional promoter polymorphism in the macrophage migration inhibitory factor (MIF) gene associated with disease severity in rheumatoid arthritis. Genes Immun. 3:170-176.

2. Burger-Kentischer, A., et al. 2002. Expression of macrophage migration inhibitory factor in different stages of human atherosclerosis. Circulation. 105:1561-1566.

3. Calandra, T., et al. 2000. Protection from septic shock by neutralization of macrophage migration inhibitory factor. Nat. Med. 6:164-170.

4. Calandra, T., et al. 1995. MIF as a glucocorticoidinduced modulator of cytokine production. Nature. 377:68-71.

5. Flaster, H., Bernhagen, J., Calandra, T., and Bucala, R. 2007. The macrophage migration inhibitory factor-glucocorticoid dyad: regulation of inflammation and immunity. Mol. Endocrinol. 21:1267-1280.

6. Takahashi, M., et al. 2002. Elevation of plasma levels of macrophage migration inhibitory factor in patients with acute myocardial infarction. Am. J. Cardiol. 89:248-249.

7. Yu, C.M., Lai, K.W., Chen, Y.X., Huang, X.R., and Lan, H.Y. 2003. Expression of macrophage migration inhibitory factor in acute ischemic myocardial injury. J. Histochem. Cytochem. 51:625-631.

8. Chagnon, F., Metz, C.N., Bucala, R., and Lesur, O. 2005. Endotoxin-induced myocardial dysfunction: effects of macrophage migration inhibitory factor neutralization. Circ. Res. 96:1095-1102.

9. Benigni, F., et al. 2000. The proinflammatory mediator macrophage migration inhibitory factor induces glucose catabolism in muscle. J. Clin. Invest. 106:1291-1300.

10. Miller, E.J., et al. 2008. Macrophage migration inhibitory factor stimulates AMP-activated protein kinase in the ischaemic heart. Nature. 451:578-582.

11. Russell, R.R., 3rd, et al. 2004. AMP-activated protein kinase mediates ischemic glucose uptake and prevents postischemic cardiac dysfunction, apoptosis, and injury. J. Clin. Invest. 114:495-503.

12. Weston, C.R., and Davis, R.J. 2007. The JNK signal transduction pathway. Curr. Opin. Cell Biol. 19:142-149.

13. Yin, T., et al. 1997. Tissue-specific pattern of stress kinase activation in ischemic/reperfused heart and kidney. J. Biol. Chem. 272:19943-19950.

14. Mizukami, Y., Yoshioka, K., Morimoto, S., and Yoshida, K. 1997. A novel mechanism of JNK1 activation. Nuclear translocation and activation of JNK1 during ischemia and reperfusion. J. Biol. Chem. 272:16657-16662.

15. Kaiser, R.A., et al. 2005. Genetic inhibition or acti- vation of JNK $1 / 2$ protects the myocardium from ischemia-reperfusion-induced cell death in vivo. J. Biol. Chem. 280:32602-32608.

16. Kleemann, R., et al. 2000. Intracellular action of the cytokine MIF to modulate AP-1 activity and the cell cycle through Jab1. Nature. 408:211-216.

17. Nguyen, M.T., et al. 2003. The cytokine macrophage migration inhibitory factor reduces pro-oxidative stress-induced apoptosis. J. Immunol. 170:3337-3347.

18. Watanabe, H., et al. 2004. Ultraviolet A-induced production of matrix metalloproteinase- 1 is mediated by macrophage migration inhibitory factor (MIF) in human dermal fibroblasts. J. Biol. Chem. 279:1676-1683.

19. Dhanantwari, P., et al. 2008. Macrophage migration inhibitory factor induces cardiomyocyte apoptosis. Biochem. Biophys. Res. Commun. 371:298-303.

20. Hausenloy, D.J., and Yellon, D.M. 2006. Survival kinases in ischemic preconditioning and postconditioning. Cardiovasc. Res. 70:240-253.

21. Shao, Z., et al. 2006. c-Jun $\mathrm{N}$-terminal kinases mediate reactivation of Akt and cardiomyocyte survival after hypoxic injury in vitro and in vivo. Circ. Res. 98:111-118.

22. Leng, L., et al. 2003. MIF signal transduction initiated by binding to CD74. J. Exp. Med. 197:1467-1476.

23. Garner, L.B., et al. 2003. Macrophage migration inhibitory factor is a cardiac-derived myocardial depressant factor. Am. J. Physiol. Heart Circ. Physiol. 285:H2500-H2509.

24. Qi, X., Vallentin, A., Churchill, E., and MochlyRosen, D. 2007. deltaPKC participates in the endoplasmic reticulum stress-induced response in cultured cardiac myocytes and ischemic heart. J. Mol. Cell. Cardiol. 43:420-428.

25. Aoki, H., et al. 2002. Direct activation of mitochondrial apoptosis machinery by c-Jun N-terminal kinase in adult cardiac myocytes. J. Biol. Chem 277:10244-10250.

26. Bennett, B.L., et al. 2001. SP600125, an anthrapyrazolone inhibitor of Jun N-terminal kinase. Proc Natl. Acad. Sci. U. S. A. 98:13681-13686.

27. Heidbreder, M., Naumann, A., Tempel, K., Dominiak, P., and Dendorfer, A. 2008. Remote vs. ischaemic preconditioning: the differential role of mitogen-activated protein kinase pathways. Cardiovasc. Res. 78:108-115.

28. Schulz, E., et al. 2008. Suppression of the JNK pathway by induction of a metabolic stress response prevents vascular injury and dysfunction. Circulation. 118:1347-1357.

29. Willis, M.S., et al. 2005. Macrophage migration inhibitory factor mediates late cardiac dysfunction after burn injury. Am. J. Physiol. Heart Circ. Physiol. 288:H795-H804.

30. Pakozdi, A., et al. 2006. Macrophage migration inhibitory factor: a mediator of matrix metalloproteinase- 2 production in rheumatoid arthritis. Arthritis Res. Ther. 8:R132.

31. Santini, E., et al. 2008. Effects of different LDL particles on inflammatory molecules in human mesangial cells. Diabetologia. 51:2117-2125.

32. Bogoyevitch, M.A., et al. 1996. Stimulation of the stress-activated mitogen-activated protein kinase subfamilies in perfused heart. p38/RK mitogenactivated protein kinases and c-Jun $\mathrm{N}$-terminal kinases are activated by ischemia/reperfusion. Circ. Res. 79:162-173.

33. Zhao, J., Pei, D.S., Zhang, Q.G., and Zhang, G.Y. 2007. Down-regulation Cdc42 attenuates neuronal apoptosis through inhibiting MLK3/JNK3 cascade during ischemic reperfusion in rat hippocampus. Cell Signal. 19:831-843.

34. Zhang, Q.G., et al. 2006. Akt inhibits MLK3/JNK3 signaling by inactivating Rac1: a protective mechanism against ischemic brain injury. J. Neurochem. 98:1886-1898.

35. Zhang, Q.G., et al. 2005. Knock-down of POSH expression is neuroprotective through down-regulating activation of the MLK3-MKK4-JNK pathway following cerebral ischaemia in the rat hippocampal CA1 subfield. J. Neurochem. 95:784-795.

36. Watanabe, T., et al. 2005. Apoptosis signal-regulating kinase 1 is involved not only in apoptosis but also in non-apoptotic cardiomyocyte death. Biochem. Biophys. Res. Commun. 333:562-567.

37. Frazier, D.P., et al. 2007. PKC-alpha and TAK-1 are intermediates in the activation of c-Jun NH2terminal kinase by hypoxia-reoxygenation. Am.J. Physiol. Heart Circ. Physiol. 292:H1675-1684.

38. Dhanasekaran, D.N., and Reddy, E.P. 2008. JNK signaling in apoptosis. Oncogene. 27:6245-6251.

39. Mizue, Y., et al. 2005. Role for macrophage migration inhibitory factor in asthma. Proc. Natl. Acad. Sci. U. S. A. 102:14410-14415.

40. Plant, B.J., et al. 2005. Cystic fibrosis, disease severity, and a macrophage migration inhibitory factor polymorphism. Am. J. Respir. Crit. Care Med. 172:1412-1415

41. Bernhagen, J., et al. 2007. MIF is a noncognate ligand of CXC chemokine receptors in inflammatory and atherogenic cell recruitment. Nat. Med. 13:587-596.

42. Das, S., Otani, H., Maulik, N., and Das, D.K. 2006. Redox regulation of angiotensin II preconditioning of the myocardium requires MAP kinase signaling. J. Mol. Cell. Cardiol. 41:248-255. 